\title{
Alternate timelines
}

Cite as: CMAJ 2020 July 27;192:E866-7. doi: 10.1503/cmaj.200124

$\mathrm{T}$ he telephone call that announced your arrival in the emergency department dragged me out of a deep, deep sleep. It was my second overnight shift of the weekend as a junior internal medicine resident, and fatigue was starting to set in. Even after I splashed icy water on my face and trudged to the emergency department, the fog had not completely cleared. I caught only the relevant snippets as the emergency doctor filled me in.

"Advanced cancer." "Months left to live." "Became suddenly confused overnight." "Now completely unresponsive." "Family understands and doesn't want any heroic acts."

And the grand finale:

"He just needs admission to hospital to be palliated at the end of life."

My mind latched onto this last sentence. Suddenly, you were no longer a complex patient with multiple medical issues that needed attention; you were a man who wanted to be comfortable in your imminent death. Simple.

I walked into your room, introduced myself to your family and re-outlined the plan suggested by the emergency doctor. Your family members exchanged indecipherable glances.

"Well, you see," your brother ventures. "We were just talking and we're actually not sure anymore ..."

Just like that, the story that had been spun so neatly about you began to unravel.

I scanned the room, taking stock of your rapid heart rate, falling blood pressure, the high-flow oxygen mask strapped to your face. Suddenly, all these details were potentially relevant again. I became acutely aware of how little time we had to make a decision and act on it.

I felt intensely conflicted and reached for what I thought was the right answer the one that fit the narrative I had originally been told. I reiterated that treating

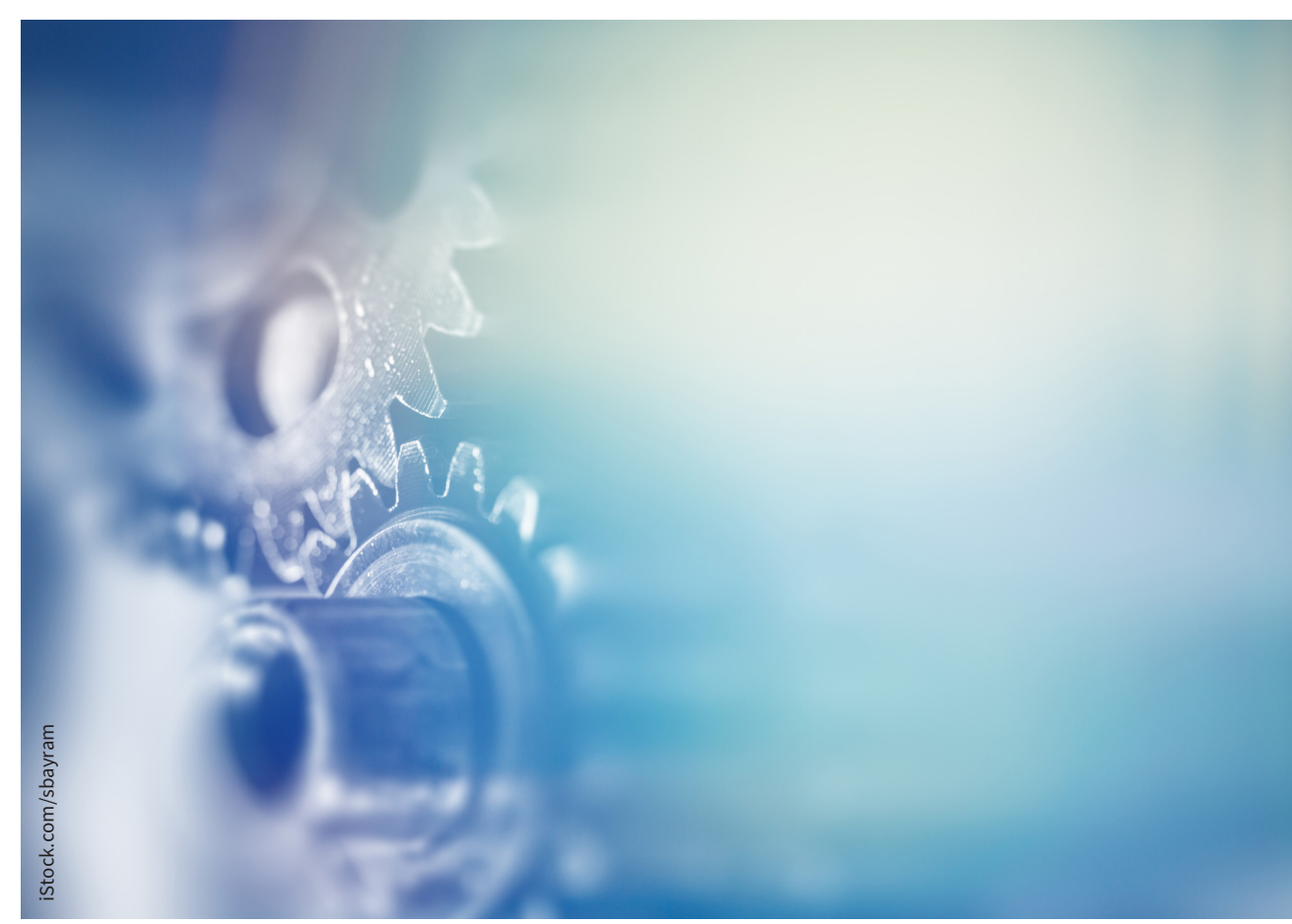

aggressively would be at the expense of comfort, which would be cruel if someone had already decided they wanted to go peacefully.

Your family paused before replying. They agreed that they didn't want to create unnecessary suffering through chest compressions or electric shocks, but what if there were other options that didn't cause such blatant harm? They revealed that you had actually been reasonably well before things suddenly went downhill; that a week ago you were helping here and there with the family business, albeit between bouts of severe nausea. Perhaps you would have wanted active treatment against an acute illness like this one.

I felt frustrated about this situation, which was slipping further and further away from my initial expectations. I had made the false assumption that we were confident about what you wanted, and this assumption had carried an inertia that made it difficult for me to let go. Now, in the absence of that certainty, your future gaped like a vast, unknowable chasm. I studied your still face, desperately hoping to find an answer. There was none.

"Okay. We can try a few things," I finally ventured. "But even if this works, there's no guarantee he will regain his previous functional status. Do you think this is what he would have wanted?"

More glances were exchanged, and then your brother spoke up.

"At this point, we don't know. He has told us before that he doesn't want CPR and he doesn't want to suffer needlessly. But that doesn't necessarily tell us what he would want in this exact situation, does it?"

He paused before speaking again, this time with a little more confidence. "I think he would at least want to try medical therapy." 
"Right. Okay."

This was not the conclusion I was expecting, but I was too tired to discuss it any further. I ordered an anti-arrhythmic infusion through your IV.

What happened next appeared to be nothing short of a small miracle. Immediately, your heart rate settled. You flipped from comatose to conversational within thirty minutes.

Two days later, you were giving me input on how to advance your care. "You should decrease the fluids I'm getting through this IV," you suggested, "since I'm drinking better now. Plus, my hand is getting swollen."

Smiling, I responded, "That's exactly what I was planning to do."

That same week, you got your first dose of palliative radiation. Your response was so good that your oncologist extended your predicted lifespan.

You ate meals. You stood up and walked, albeit with difficulty. You left the hospital and returned home to your family.

Looking back to that night, I see with startling clarity how close we came to making a decision to stop active treatment and palliate. A decision that, with hindsight, now felt like it would have been the wrong one. The decision belonged to your family, but I was the medical professional standing in the room guiding them. If I had pushed them harder toward palliation, and they had agreed, I would have denied you those extra weeks to months with your loved ones. You almost died that night, mostly because you were objectively very sick and dying, but also because we very nearly decided not to fix that.

When I think back to what happened, I imagine two possible timelines that diverged when we decided to attempt to resuscitate you. I recall similar patients, slowly dying people who, when faced with a crisis, wanted comfort over prolonging further suffering. In those situations, after we had unanimously agreed upon what was best, we had never doubted or even glanced back. There had been no opportunity for an alternate timeline to appear.

After seeing what happened with you, I couldn't help scanning these memories again, wondering which patients I could have "saved" - or at least given a few more good weeks - if we had made different choices. Against my better judgment, I find myself haunted by the unanswerable questions that these alternate timelines pose.

Of course, I soon realized this was a futile exercise, trying to judge these decisions as "right" or "wrong" based on theoretical events of the future, whether the immediate outcomes or the potentially long roads that lead from them. In medicine, we are not granted the gifts of precognition and omniscience. We, alongside our patients, make life-changing decisions using only the limited information we have in our hands. The truth is that there is only ever one timeline. We can do only our best by our patients, and we hold out hope not for the "right" choice but for a good outcome - in whatever form it may take.

\section{Grace Zhang MD}

Queen's University, Kingston, Ont.

This article has been peer reviewed.

This is a true story. The patient's family has given consent for this story to be told. 